\section{Interim PET in Hodgkin Lymphoma: Is It So Useless?}

TO THE EDITOR: We read with interest the above letter from Adams and Kwee when it was published online ahead of print, and we think it important to comment on the strong and recognized impact of interim PET in the management of Hodgkin lymphoma. More than 2,000 patients with advanced disease were included in 3 well-designed prospective trials, in which patients who-after 2 cycles of ABVD (doxorubicin, bleomycin, vinblastine, dacarbazine)-were PET-positive (Deauville score $\geq 4$ ) were escalated to BEACOPP (bleomycin, etoposide, adriamycin, cyclophosphamide, vincristine, procarbazine, and prednisone) whereas those who were PET-negative continued with the standard regimen (4 cycles of ABVD) (1-3). The PET positivity rate using this Deauville score as the cutoff was similar across these 3 studies, as was the outcome of both PET-positive and PET-negative patients. The study of the Southwest Oncology Group (1) enrolled 336 patients, 18\% of whom were PET-positive. The "Response Adapted Therapy in Advanced Hodgkin Lymphoma" study (2) enrolled 1,119 patients, $25 \%$ of whom were PET-positive. The Italian GITIL/FIL HD0607 trial (3) enrolled 773 patients, $19 \%$ of whom were PET-positive. Overall, the 2-y progression-free survival of the PET-positive patients ranged between $64 \%$ and $67 \%$. PET-negative patients who followed the standard regimen had a 2-y progression-free survival ranging between $82 \%$ and $89 \%$, higher than the progression-free survival of the whole population.

A major advantage of this strategy is that PET-negative patients ( $>80 \%$ of the total population) can be spared from the adverse effects of BEACOPP. As pointed out by Adams and Kwee, none of the interim ${ }^{18} \mathrm{~F}$-FDG PET/CT-adapted trials that have been performed so far had a control arm; that is, none continued standard ABVD in PET-positive patients. Apart from the fact that this arm would have been ethically difficult to defend, series have found 2-y progression-free survivals of $12 \%-27 \%$ in advanced-stage Hodgkin lymphoma patients PET-positive after 2 ABVD cycles-much lower than the $64 \%-67 \%$ found when these patients were intensified with BEACOPP (4,5), with this strategy therefore clearly having an advantage in improving outcome and decreasing the number of events. This finding was further confirmed by the results of the EORTC/LYSA/FIL H10 trial (6), which included 1,950 patients with early-stage Hodgkin lymphoma randomized between an experimental arm and a standard arm. Patients PET-positive after 2 ABVD cycles had a 5-y progression-free survival of $77.4 \%$ in the experimental arm that received standard ABVD plus involved-node radiotherapy, and survival improved to $90.6 \%$ in the experimental arm that received BEACOPP escalation plus involved-node radiotherapy (hazard ratio, $0.42 ; 95 \%$ confidence interval, $0.23-0.74 ; P=0.002$ ).

A deescalation trial (7) in 823 patients with advanced Hodgkin lymphoma (AHL2011 LYSA trial) has also recently confirmed the major role of an interim PET-guided strategy. Patients PETnegative after 2 BEACOPP escalations were moved to ABVD. Their progression-free survival was not inferior to that of the standard arm, in which BEACOPP was continued. Thus, deescalation avoids the toxic effect of BEACOPP while producing the same outcome.

In view of the cited evidence, we strongly disagree with Adams and Kwee and support the recommended role of interim PET in
Hodgkin lymphoma management, which allows better tailoring of treatment to individual patients $(8)$.

\section{REFERENCES}

1. Press OW, Li H, Schoder H, et al. US intergroup trial of response-adapted therapy for stage III to IV Hodgkin lymphoma using early interim fluorodeoxyglucosepositron emission tomography imaging: Southwest Oncology Group S0816. J Clin Oncol. 2016;34:2020-2027.

2. Johnson P, Federico M, Kirkwood A, et al. Adapted treatment guided by interim PET-CT scan in advanced Hodgkin's lymphoma. N Engl J Med. 2016;374:24192429 .

3. Gallamini A, Rossi A, Patti C, et al. Interim PET-adapted chemotherapy in advanced Hodgkin lymphoma (HL): results of the second interim analysis of the Italian GITIL/FIL HD0607 trial [abstract]. Hematol Oncol. 2015;33 (suppl): 163 .

4. Gallamini A, Hutchings M, Ritacco L, et al. Early interim 2-[ $\left[{ }^{18} \mathrm{~F}\right]$ fluoro-2-deoxyD-glucose positron emission tomography is prognostically superior to international prognostic score in advanced-stage Hodgkin's lymphoma: a report from a joint Italian-Danish study. J Clin Oncol. 2007;25:3746-3752.

5. Gallamini A, Barrington SF, Biggi A, et al. The predictive role of interim positron emission tomography for Hodgkin lymphoma treatment outcome is confirmed using the interpretation criteria of the Deauville five-point scale. Haematologica. 2014;99:1107-1113.

6. Andre MP, Girinsky T, Federico M, et al. Early positron emission tomography response-adapted treatment in stage I and II Hodgkin lymphoma: final results of the randomized EORTC/LYSA/FIL H10 trial. J Clin Oncol. March 14, 2017 [Epub ahead of print]

7. Casasnovas O, Brice P, Bouabdallah R, et al. Randomized phase III study comparing an early PET driven treatment de-escalation to a not PET-monitored strategy in patients with advanced stages Hodgkin lymphoma: interim analysis of the AHL2011 Lysa study [abstract]. Blood. 2015;126:577.

8. Johnson PW. Response-adapted frontline therapy for Hodgkin lymphoma: are we there yet? Hematology Am Soc Hematol Educ Program. 2016;2016:316-322.

$\begin{array}{r}\text { Michel Meignan* } \\ \text { Anne Ségolène Cottereau } \\ \text { Bénédicte Deau } \\ \text { Salim Kanoun }\end{array}$
Blina Berriolo-Riedinger
Olivier Casasnovas
*Hospital Henri Mondor
51, avenue du Marechal de Lattre de Tassigny
Creteil, 94010, France
E-mail: Michel.meignan@aphp.fr

Published online Mar. 2, 2017.
DOI: $10.2967 /$ jnumed.117.190462

REPLY: We thank Meignan et al. for their interest in our letter, in which we document the limitations and low necessity of interim ${ }^{18}$ F-FDG PET/CT imaging in lymphoma. Although Meignan et al. seem to agree with us that interim ${ }^{18}$ F-FDG PET/CT has low clinical value in non-Hodgkin lymphoma $(1,2)$, they disagree on the value of interim ${ }^{18} \mathrm{~F}$-FDG PET/CT in Hodgkin lymphoma, which we will therefore discuss in this reply.

Hodgkin lymphoma is usually divided into early- and advancedstage disease, which are treated differently and have a different prognosis. Studies have shown that the value of interim ${ }^{18} \mathrm{~F}-\mathrm{FDG}$ PET/CT for predicting outcome is not homogeneous in these different disease entities. In early-stage Hodgkin lymphoma, the value of interim ${ }^{18} \mathrm{~F}$-FDG PET/CT can be considered low: patients with positive interim ${ }^{18} \mathrm{~F}$-FDG PET/CT findings have been reported to have a generally good progression-free survival (range, 